\title{
Degradation and Physical Properties of Aliphatic Copolyesters Derived from Mixed Diols
}

\author{
Youngtai Yoo, ${ }^{\dagger}$ Myung-Suk Ko, Sang-Il Han, Tae-Yoon Kim, \\ Seungsoon IM, ${ }^{*}$ and Dong-Kook KIM** \\ Department of Industrial Chemistry, College of Engineering, Kon Kuk University, \\ 93-1 Mojin-dong, Kwangjin-ku, Seoul, Korea 143-701 \\ * Department of Textile Engineering, Hanyang University, Seoul, Korea \\ ** Department of Chemistry, Hanyang University, Ansan, Korea
}

(Received July 7, 1997)

\begin{abstract}
The effects of molecular structure and composition of poly(butylene succinate) (4-4 polyester derived from succinic acid and 1,4-butane diol) rich random copolyesters on the physical properties and hydrolytic degradation were investigated. Three series of copolyesters, poly(butylene succinate-co-ethylene succinate), poly(butylene succinate-co-propylene succinate), and poly(butylene succinate-co-butyne succinate) were synthesized using ethylene glycol (EG), propylene glycol (PG), and cis-2-butene-1,4-diol (BeD) as a comonomer, respectively up to $40 \mathrm{~mol} \%$ of diols in the feeds in combination with 1,4-butane diol (BD) and succinic acid (SA). The copolymers were characterized for composition, inherent viscosity, molecular weight, density, thermal properties, and mechanical strength. The melting temperature of copolymers decreased almost linearly at the rate of $1.2-1.6 \mathrm{~K} \mathrm{~mol}^{-1}$ of ethylene succinate and propylene succinate in the test range. Remarkable improvements in ultimate strain were attained through the copolymerization using EG or PG as a comonomer. It was found that the hydrolytic degradation behaviors of copolyesters conducted in $\mathrm{pH} 12$ alkali solution were greatly affected by the chemical structure and composition of copolymers. The degradation rate of poly(butylene succinate-co-ethylene succinate) consistently increased with the content of ethylene succinate, whereas poly(butylene succinate-co-butyne succinate) showed an adverse effect. The copolymers comprising propylene succinates showed a composition dependancy on the hydrolytic susceptability ascribable to the conflicting effects between steric hinderance due to methyl substituents and reduced crystallinity.
\end{abstract}

KEY WORDS Aliphatic Polyester/ Poly(butylene succinate)/ Copolymerization/ Physical Properties/ Hydrolytic Degradation/Crystallinity/ Topology/Substituent Effect /

It has been widely recognized that aliphatic polyesters, in general, undergo complete degradation by hydrolysis and/or by enzymatic attack within a reasonable time frame unlike their aromatic counterparts. ${ }^{1-3}$ Poly(butylene succinate), (PBS hereafter) is one of the most viable biodegradable polymers which may replace many conventional plastics in near future for having acceptable mechanical strength and comparable softening temperature to low density polyethylene and polystyrene. ${ }^{4} \mathrm{Re}-$ cently, poly(butylene succinate), poly(butylene adipate) and their copolyesters have been produced on commercial basis. Academic and industrial interests have been directed towards the studies on structure, properties, and biodegradation behavior of these materials for the growing importance of environmentally degradable polymers. ${ }^{5-8}$

There are a number of structural factors which affect the biodegradability of aliphatic polyesters. One of the most critical parameters of biodegradation may be the crystalline structure. ${ }^{9}$ It is generally conceived that the incorporation of secondary repeating unit along the polyester chain reduces the crystallinity of parent polymer by disturbing the regularity of chemical structure and hence the lateral packing of its crystallites. ${ }^{10,11} \mathrm{On}$ the other hand, isomorphism is frequently reported in linear aliphatic copolyesters such as poly(hexamethylene adipate-co-hexamethylene sebacate) and poly(decamethylene adipate-co-decamethylene sebacate) ${ }^{12,13}$ and poly(3-hydroxy butylate-co-3-hydroxyvalerate). ${ }^{14}$

In a practical sense, it seems very crucial to develop

† To whom correspondence should be addressed. aliphatic polyester systems with a wide spectrum of degradation rate to promote their applications. Accordingly, modification of the chemical structure of aliphatic polyesters with the purposes of degradation control and improving critical physical properties has been one of the intriguing subjects in this field.

The present paper deals with syntheses, characterization, and hydrolytic degradation behavior of three classes of PBS rich random copolymer systems prepared by employing limited amounts of comonomers, including ethylene glycol (EG), propylene glycol (PG), and cis2-butene-1,4-diol (BeD). It was visioned that each diol might introduce unique structural variation to PBS homopolymer with regards to crystalline structure, steric and topological aspects. For instance, propylene glycol will result in copolyester with alkyl substituents. Furthermore, cis-2-butene diol may generate considerable irregularity along the all trans planar zigzag conformation of polyester. The effects of chemical modification of PBS by copolymerization using various diols on the physical properties and hydrolytic degradation will be discussed.

\section{EXPERIMENTAL}

\section{Material and Polymerization}

Succinic acid, butane diol, EG, PG (Junsei Chem.), and $\mathrm{BeD}$ (Aldrich Chem. Co.) were used without further purification. Titanium tetrabutoxides (TTB) was obtained from Aldrich and used as received. PBS and copolyesters were prepared employing two-step polymerization route. ${ }^{15}$ First, calculated amounts of succinic 
acid and diols ( $1: 1.2$ by mole ratio) were introduced to a reactor equipped with an overhead stirrer and a temperature control device. The monomer mixture was melted and stirred at $190^{\circ} \mathrm{C}$ for $2 \mathrm{~h}$. Evolved water was removed passing through a condensation column. Then, TTB catalyst $(0.3 \mathrm{wt} \%$ of monomer $)$ was charged to the reactor and the reaction temperature was raised to $240^{\circ} \mathrm{C}$ over a period of $30 \mathrm{~min}$ gradually applying a reduced pressure to remove low molecular weight condensates and maintained for $2-7 \mathrm{~h}$ depending on the feed composition.

\section{Sample Preparation and Measurements}

In order to provide identical samples and to ensure a consistency in various measurements synthesized polymers were compression molded using a Carver Press at $150^{\circ} \mathrm{C}$ and 10000 psi. The molded films were air cooled and stored at room temperature for at least $24 \mathrm{~h}$ before any measurements. Thickness of the molded films was controlled by using a stainless steel spacer $(0.3 \mathrm{~mm}$ thick $)$ placed between hot plates of the Carver Press.

${ }^{1} \mathrm{H}$ NMR spectra were recorded on a Bruker $500 \mathrm{MHz}$ spectrometer. Specific gravity of each polymer was measured using a density gradient column filled with $\mathrm{CCl}_{4}$ and $n$-heptane. Molecular weight and polydispersity were determined from gel permeation chromatography (Waters) equipped with styragel column and RI detector using polystyrene standards. A mixture of $\mathrm{CHCl}_{3}$ and $o$-chlorophenol (4: 1 by vol.) was used as a solvent system. Inherent viscosities of polymers were measured in a Cannon-Ubbelohde viscometer at $25^{\circ} \mathrm{C}$ in chloroform solution of $1 \mathrm{~g} \mathrm{dl}^{-1}$ concentration. Thermal analysis of the sample was performed on a DSC(TA Instruments) with a heating and cooling rate of $10^{\circ} \mathrm{C} \mathrm{min}^{-1}$. The crystalline structure of PBS and copolyesters were examined using X-ray diffractomenter (Rigaku Denki Co.) equipped with a monochrometer and graphite crystal $\left(\mathrm{Cu}-K_{\alpha}\right.$ radiation). Mechanical properties of polymers were evaluated also on the dumbell shape film with a dimension of $3.5 \mathrm{~mm}$ width, $14 \mathrm{~mm}$ gauge length, and $0.3 \mathrm{~mm}$ thickness from universal test machine (Instron) with $5 \mathrm{kN}$ load cell and the grip distance of $26 \mathrm{~mm}$ at the cross head speed of $20 \mathrm{~mm} \mathrm{~min}^{-1}$.

\section{Degradation Study}

Hydrolytic degradation of polyester was studied on compression molded film in a $\mathrm{NaOH}$ basic aqueous solution of $\mathrm{pH} 12$ at $35^{\circ} \mathrm{C}$. Each film was immersed in a separate erlenmayer flask which was placed in a temperature controlled incubator at a shaking speed of $45 \mathrm{rpm}$. Each sample was periodically taken from the solution and was carefully weighed after drying under a reduced pressure.

\section{Sample Identification}

Poly(butylene succinate-co-ethylene succinate), poly(butylene succinate-co-propylene succinate), and poly(butylene succinate-co-butyne succinate) were abbreviated to poly(BS-co-ES), poly(BS-co-PS), and poly(BS-co$\mathrm{BeS})$, respectively. The numbers at the end of these codes denote the mole fractions of minor species such as ES, $\mathrm{PS}$, and BeS. For example, poly(BS-co-ES)-26 represents poly(butylene succinate-co-ethylene succinate) contain- ing $74 \mathrm{~mol} \%$ of butylene succinate and $26 \mathrm{~mol} \%$ of ethylene succinate.

\section{RESULTS AND DISCUSSION}

\section{Polymer Synthesis and Characterization}

PBS and copolyesters were synthesized via two-step process consisted of direct esterification and polycondensation. The first stage of polymerization was carried out under atmospheric pressure to form oligomeric species. The number-average molecular weight of oligomer was in the range of $900-1200 \mathrm{~g} \mathrm{~mol}^{-1}$ (by GPC, $\left.X_{n}=5-7\right)$ after the direct esterification step for $2 \mathrm{~h}$. Then, the polycondensation of oligomers was proceeded at $240^{\circ} \mathrm{C}$ under a reduced pressure, where titanium tetrabutoxide was introduced to the reaction mixture as a catalyst. The results of polyester syntheses are summarized in Table I. As shown in the table it was possible to synthesize the copolyesters with comparable molecular weights so that the effect of molecular weight on the physical properties and biodegradation may be excluded. It was found that polydispersities of the polymers were found to be slightly higher than typical value in step growth polymerization. However, poly(BSco-BeS) showed broader molecular weight distribution as content of unsaturated butyne succinate unit increased. In order to obtain comparable molecular weight to PBS homopolymer longer reaction time was allowed for the feeds containing ethylene glycol or propylene glycol for their lower reactivities than butane diol. It may be due to the change in reactivity of remaining hydroxyl group upon reaction of the first one, occurring with the diols having two functional groups in close proximity. ${ }^{16}$ Esterification involves a significant change in the electron-donating ability of the functional group. The reactivity of ethylene glycol towards esterification is considerably higher than half-esterified glycol because an adjacent ester group has less electron donation ability than hydroxyl group. ${ }^{17}$ In addition, the presence of secondary hydroxyl group in propylene glycol should be responsible for the slower polymerization. In contrast, melt viscosity of reaction mixture increased fast when butene diol was used as a comonomer. The electron rich double bond in the monomer may have promoted the nucleophilicity of hydroxyl groups. However, cross polymerization via carbon-carbon double bond could be the more plausible explanation for the rapid increase in melt viscosity during the polycondensation at such an elevated temperature.

${ }^{1} \mathrm{H}$ NMR study indicates that the copolyesters contain substantially less amounts of minor species than the corresponding feed compositions, especially in poly(BS-co-PS)s. The higher volatility as well as the lower reactivity of $E G$ or $P G$ may be the reasons for such differences between feed and polymer compositions. However, it was rather surprising to note that the poly(BS-co-BeS) series also have considerably less amounts of minority constituent, butyne succinate than feeds as calculated from the integration of methine $(\delta: 4.70 \mathrm{ppm})$ and adjacent methylene groups $(\delta: 4.11 \mathrm{ppm})$ in the NMR spectra. A part of the discrepancy between feeds and polymer compositions may be due to the cross polymerization which consume some of unsaturated moieties 
Y. Yoo et al.

Table I. Polymerization data and properties of PBS and copolyesters

\begin{tabular}{|c|c|c|c|c|c|c|c|}
\hline \multirow{2}{*}{ Polymer code } & \multicolumn{2}{|c|}{ Molar composition (BD : $\mathrm{SD}^{\mathrm{a}}$ ) } & \multirow{2}{*}{$\frac{\text { Reaction time }}{\mathrm{h}}$} & \multirow{2}{*}{$\frac{[\eta]^{\mathrm{c}}}{\mathrm{dlg}^{-1}}$} & \multirow{2}{*}{$\frac{\rho^{\mathrm{d}}}{\mathrm{g} \mathrm{cm}^{-3}}$} & \multirow{2}{*}{$\frac{\bar{M}_{n}^{\mathrm{e}} \times 10^{-3}}{\mathrm{~g} \mathrm{~mol}^{-1}}$} & \multirow{2}{*}{$\bar{M}_{w} / \bar{M}_{n}$} \\
\hline & $\begin{array}{c}\text { Feed } \\
\text { composition }\end{array}$ & $\begin{array}{c}\text { Observed } \\
\text { composition }^{b}\end{array}$ & & & & & \\
\hline PBS & $100 / 0$ & $100 / 0$ & $2+4$ & 1.3 & 1.2760 & 6.2 & 2.54 \\
\hline $\mathrm{P}(\mathrm{BS}-\mathrm{co}-\mathrm{ES})-8$ & $90 / 10$ & $92 / 8$ & $2+4$ & 1.3 & 1.2792 & 6.4 & 2.77 \\
\hline $\mathrm{P}(\mathrm{BS}-c o-\mathrm{ES})-26$ & $70 / 30$ & $74 / 26$ & $2+5$ & 1.0 & 1.2857 & 5.3 & 2.77 \\
\hline $\mathrm{P}(\mathrm{BS}-\mathrm{co}$-ES)-36 & $60 / 40$ & $64 / 36$ & $2+6$ & 1.0 & 1.2899 & 5.1 & 2.93 \\
\hline $\mathrm{P}(\mathrm{BS}-c o-\mathrm{PS})-4$ & $90 / 10$ & $96 / 4$ & $2+4$ & 1.2 & 1.2739 & 6.1 & 2.75 \\
\hline P(BS-co-PS)-9 & $80 / 20$ & $91 / 9$ & $2+5$ & 1.4 & 1.2737 & 5.8 & 2.41 \\
\hline $\mathrm{P}(\mathrm{BS}-c o-\mathrm{PS})-18$ & $70 / 30$ & $82 / 18$ & $2+6$ & 1.3 & 1.2710 & 5.0 & 2.62 \\
\hline $\mathrm{P}(\mathrm{BS}-c o-\mathrm{PS})-26$ & $60 / 40$ & $74 / 26$ & $2+7$ & 1.1 & 1.2668 & 4.9 & 2.78 \\
\hline $\mathrm{P}(\mathrm{BS}-\mathrm{co}-\mathrm{BeS})-3$ & $95 / 5$ & $97 / 3$ & $2+2$ & 1.3 & 1.2727 & 6.1 & 3.37 \\
\hline $\mathrm{P}(\mathrm{BS}-c o-\mathrm{BeS})-7$ & $90 / 10$ & $93 / 7$ & $2+2$ & 1.4 & 1.2778 & 5.1 & 5.12 \\
\hline
\end{tabular}

${ }^{a}$ Ethylene glycol, propylene glycol, and 2-butene-1,4-diol were used as second diols (SD) in synthesis. ${ }^{\mathbf{b}}$ Polymer composition was obtained from ${ }^{1} \mathrm{H}$ NMR measurement. ${ }^{c}$ Inherent viscosity determined in chloroform solution at $25^{\circ} \mathrm{C}$. ${ }^{d}$ Density was measured in a $\mathrm{CCl}_{4} / n$-heptane density gradient column. ${ }^{\mathrm{e}}$ Molecular weights were determined by GPC.

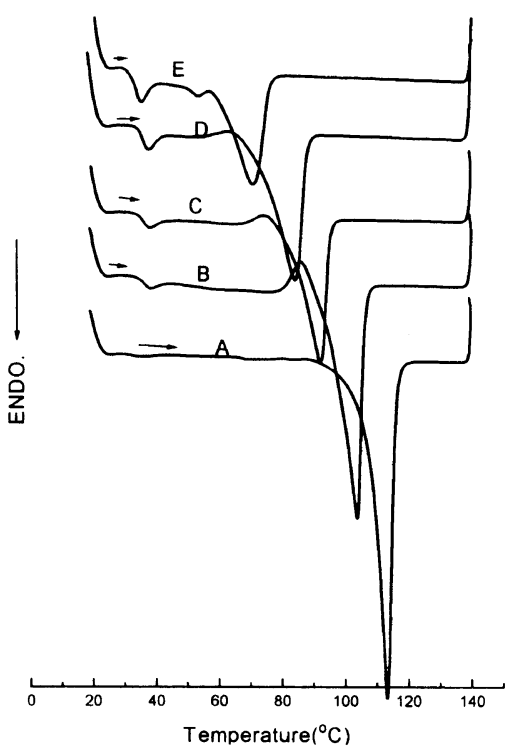

(a)

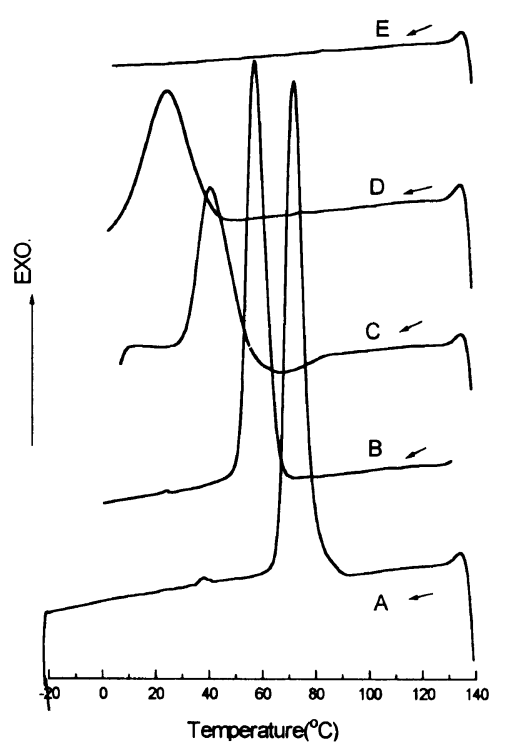

(b)

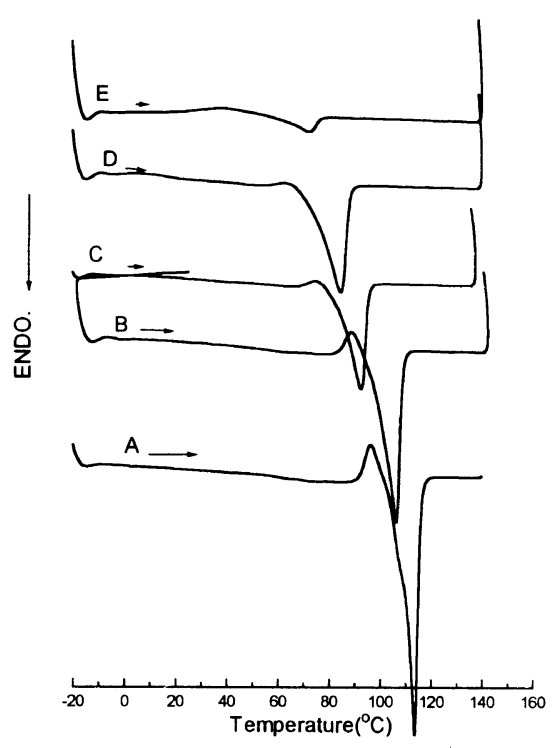

(c)

Figure 1. DSC traces of poly(BS-co-ES)s at a heating and cooling rate of $10^{\circ} \mathrm{Cmin}^{-1}$ : (a) first heating (b) cooling (c) second heating; A, PBS homopolymer; B, poly(BS-co-ES)-8; C, poly(BS-co-ES)-17; D, poly(BS-co-ES)-26; E, poly(BS-co-ES)-36.

in the chain. Sequence distribution analysis on these copolymers was not elaborated in this study since it has been well established that the step growth copolyesters derived from mixed monomers, in general, have randomly distributed sequence of respective repeating unit. $^{18,19}$

It was noted that the densities of poly(BS-co-ES) series given in Table I exhibit a consistent increase with content of ES unit, whereas an opposite trend was observed in poly(BS-co-PS)s. All the copolyesters demonstrated the lower crystallinity than PBS homopolymer. The monoclinic crystal density of poly(ethylene succinate) is reported to have significantly higher value of 1.55 than that of PBS, $1.324 .^{20}$ The dimensions of PES crystal unit cell in lateral direction is smaller, which allows more dense packing in crystalline region. Since both ES and BS units possess strictly linear structure and hence it is likely that some cocrystallization is taking place if not as quantitatively as in copolymers of 6-6 polyester and 6-10 polyester. The sluggish reduction of heat of fusion in this series compared to poly(BS-co-PS)s could be an indirect evidence. It is also possible that amorphous density could rise due to an increased polar interaction resulted from more frequent ester moieties in the copolymers than PBS homopolymer. These effect may have contributed to the density rise. For a slight increase in the density of poly(BS-co-BeS)-7 it is very unlikely that the presence of $\mathrm{BeS}$ units increase the crystal density. Only possible explanation may be due to the increase in amorphous density by intra and/or inter chain coupling based on an observation from GPC diagram, showing a distinct shoulder on high molecular side. In contrast, the presence of bulky repeating units, i.e., propylene succinate with methyl substituents in poly(BS-co-PS)s appears 


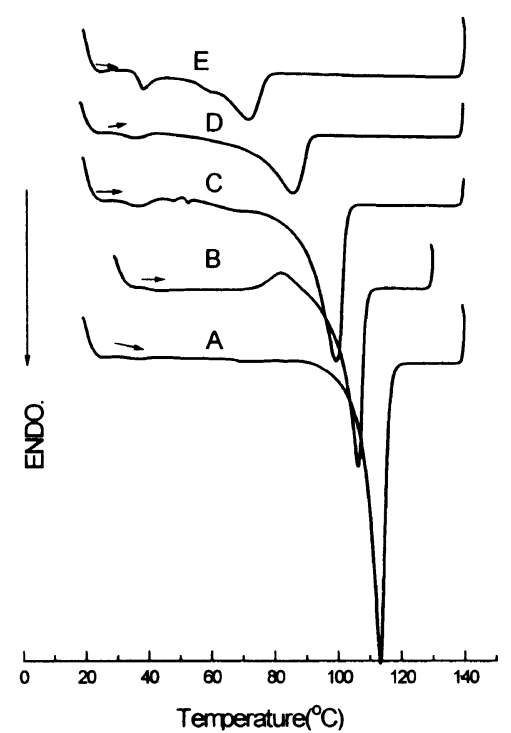

(a)

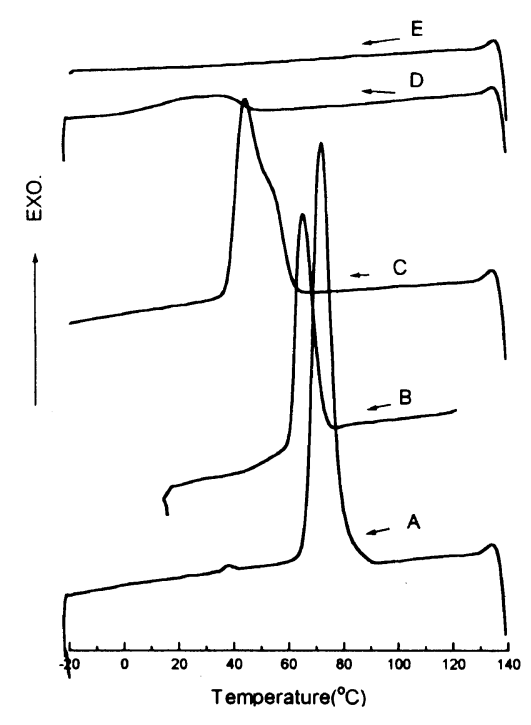

(b)

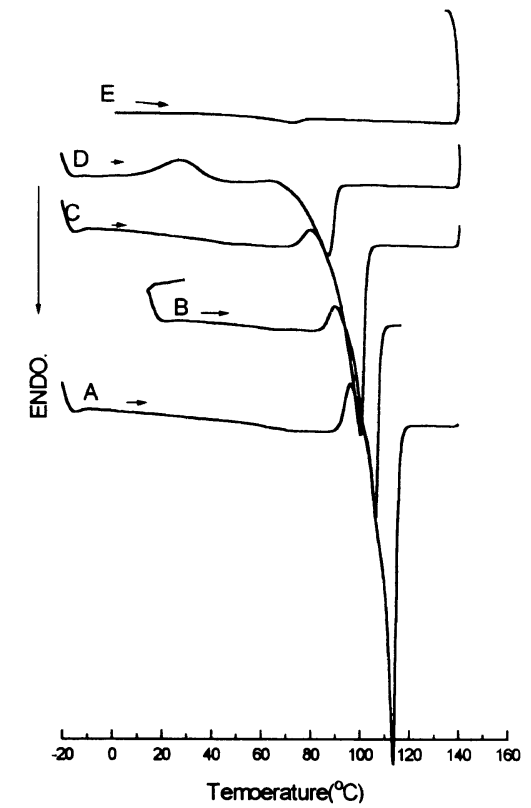

(c)

Figure 2. DSC traces of poly(BS-co-PS)s at a heating and cooling rate of $10^{\circ} \mathrm{Cmin}^{-1}$ : (a) first heating (b) cooling (c) second heating; A, PBS homopolymer; B, poly(BS-co-PS)-4; C, poly(BS-co-PS)-9; D, poly(BS-co-PS)-18; E, poly(BS-co-ES)-26.

Table II. Thermal propreties of PBS and copolyester

\begin{tabular}{|c|c|c|c|c|c|c|}
\hline \multirow{2}{*}{ Polymer code } & \multicolumn{2}{|c|}{$T_{\mathrm{m}}^{\mathrm{a}} /{ }^{\circ} \mathrm{C}$} & \multirow{2}{*}{$T_{\mathrm{c}}^{\mathrm{b} /{ }^{\circ} \mathrm{C}}$} & \multicolumn{2}{|c|}{$\Delta H_{\mathrm{f}} / \mathrm{Jg}^{-1}$} & \multirow{2}{*}{$X_{\mathrm{C}}^{\mathrm{d} / \%}$} \\
\hline & First run & Second run & & First run & Second run & \\
\hline PBS & 113.15 & 113.49 & 71.61 & 78.3 & 74.8 & 69.0 \\
\hline $\mathrm{P}(\mathrm{BS}-c o-\mathrm{ES})-8$ & 103.8 & 103.8 & 56.9 & 55.6 & 48.9 & 49.0 \\
\hline $\mathrm{P}(\mathrm{BS}-c o-\mathrm{ES})-17$ & 92.2 & 92.4 & 40.4 & 43.3 & 43.3 & 38.1 \\
\hline $\mathrm{P}(\mathrm{BS}-c o-\mathrm{ES})-26$ & 84.1 & 84.5 & 24.3 & 41.1 & 41.5 & 36.2 \\
\hline $\mathrm{P}(\mathrm{BS}-c o-\mathrm{ES})-36$ & 70.7 & 72.0 & $\mathrm{c}$ & 33.4 & 6.0 & 29.4 \\
\hline $\mathrm{P}\left(\mathrm{BS}-c^{c o-\mathrm{PS}}\right)-4$ & 106.2 & 106.2 & 64.9 & 69.2 & 69.2 & 61.0 \\
\hline P(BS-co-PS)-9 & 99.2 & 100.3 & 43.8 & 67.1 & 58.2 & 59.2 \\
\hline P(BS-co-PS)-18 & 85.7 & 87.1 & 32.3 & 37.2 & 34.2 & 32.8 \\
\hline P(BS-co-PS)-26 & 71.7 & 72.6 & c & 28.9 & 3.4 & 25.4 \\
\hline $\mathrm{P}(\mathrm{BS}-c o-\mathrm{BeS})-1.5$ & 111.9 & 112.3 & 69.2 & 74.1 & 67.9 & 65.3 \\
\hline $\mathrm{P}(\mathrm{BS}-c o-\mathrm{BeS})-3$ & 109.0 & 109.9 & 69.8 & 57.0 & 47.9 & 50.5 \\
\hline $\mathrm{P}(\mathrm{BS}-c o-\mathrm{BeS})-7$ & 106.2 & 106.4 & 64.7 & 56.7 & 45.6 & 50.0 \\
\hline
\end{tabular}

${ }^{\mathrm{a}}$ Temperature was measured at a heating rate of $10^{\circ} \mathrm{C} \min ^{-1} \cdot{ }^{\mathrm{b}} \mathrm{Temperature}$ was measured at a cooling rate of $10^{\circ} \mathrm{C} \min ^{-1}$. ${ }^{\mathrm{c}} T_{\mathrm{c}}$ was not detected at a cooling rate of $10^{\circ} \mathrm{C} \mathrm{min}^{-1} .{ }^{\mathrm{d}} X_{\mathrm{C}}$ was calculated by dividing the observed heat of fusion from the first heating trace by the theoretical value $\left(113.4 \mathrm{~J} \mathrm{~g}^{-1}\right)$ for a $100 \%$ crystalline PBS.

not to cocrystallize and thus contribute otherwise.

The thermal properties of copolyesters and PBS were measured using DSC and heating and cooling traces were collected. All the samples were heated well beyond the melting temperature and cooled back to room temperature in air so as to create uniform thermal history before the first heating scan. Figures 1 and 2 present the DSC heating and cooling traces of poly(BS-co-ES)s and poly(BS-co-PS)s, respectively. Concurrent DSC data are reported in Table II. In the DSC study of poly(BS-co-ES) and poly(BS-co-PS) series the first heating traces displayed low and high melting endotherms. The lower melting endotherms although very small in size, were found at $35-40^{\circ} \mathrm{C}$ and grew as content of ES or PS increased. Maxima of high melting endotherms shifted towards the lower side at the rate of $c a .1 .2$ and 1.6
$\mathrm{K} \mathrm{mol} \%^{-1}$ for ES and PS, respectively, whilst the shift of lower endotherms was negligible. The larger melting temperature depression in poly(BS-co-PS) series than poly(BS-co-ES)s points to the severe disruption in crystal structures which arises from the methyl substituents of propylene succinate units. The second heating traces were recorded immediately after cooling the polymer melt to ca. $-20^{\circ} \mathrm{C}$ with a cooling rate of $-10^{\circ} \mathrm{Cmin}^{-1}$. Disappreance of lower melting endotherms was noted in the second heating run, indicating that the lower melting crystals were formed at a much slower growth rate than the higher melting crystals.

The area of the major melting endotherms in DSC heating traces was integrated and reported as $\Delta H_{\mathrm{f}}$, where substantial differences in $\Delta H_{\mathrm{f}} \mathrm{s}$ from the high endotherms of first heating traces were noted among the 


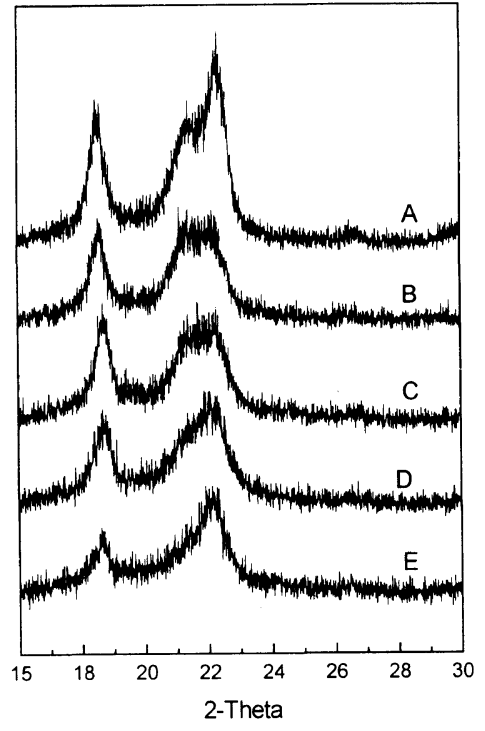

(a)

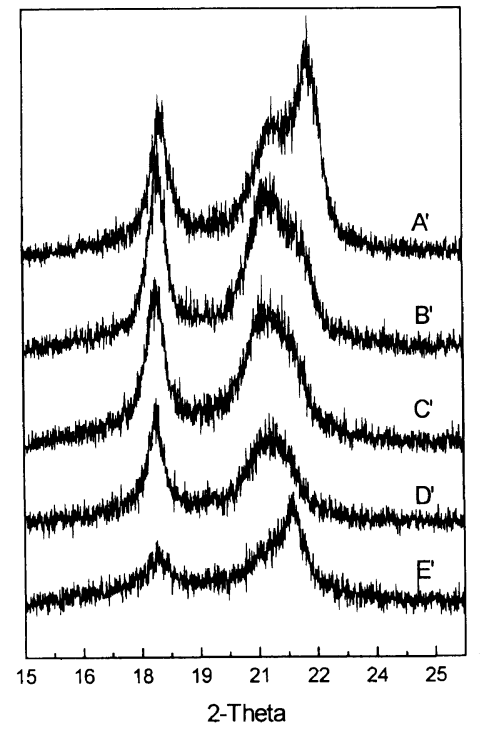

(b)

Figure 3. X-Ray diffraction patterns of poly(BS-co-ES)s (a) and poly(BS-co-PS)s (b): A, PBS homopolymer; B, poly(BS-co-ES)-8; C, poly(BS$c o$-ES)-17; D, poly(BS-co-ES)-26; E, poly(BS-co-ES)-36; A', PBS homopolymer; $\mathrm{B}^{\prime}$, poly(BS-co-PS)-4; $\mathrm{C}^{\prime}$, poly(BS-co-PS)-9; D', poly(BS-co-PS)-18; $\mathrm{E}^{\prime}$, poly(BS-co-PS)-26.

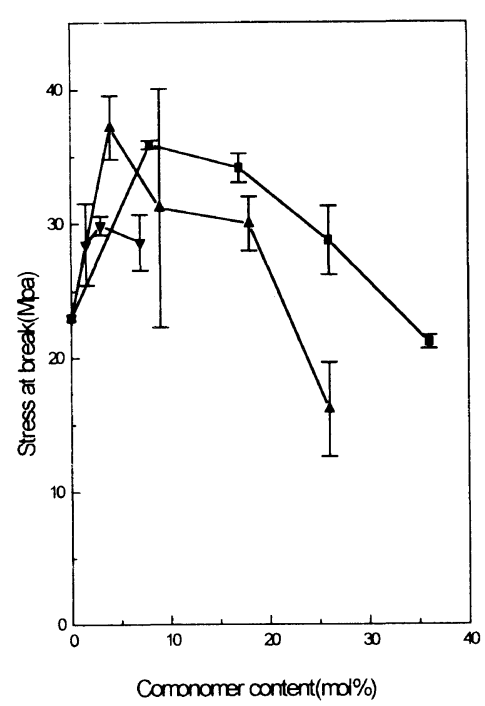

(a)

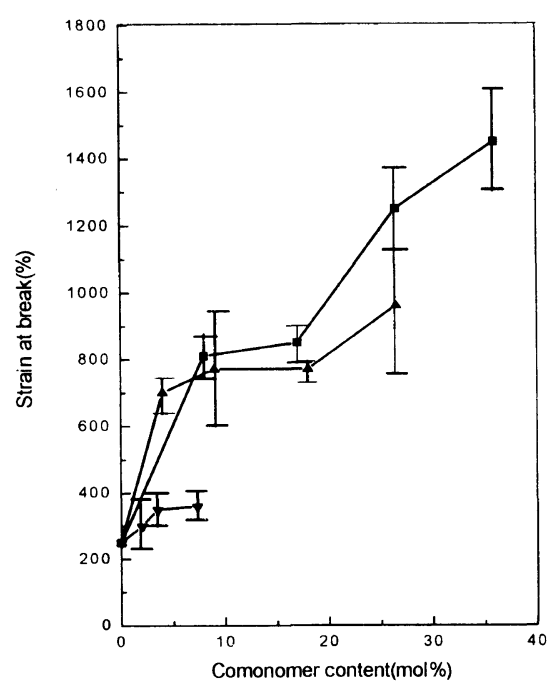

(b)

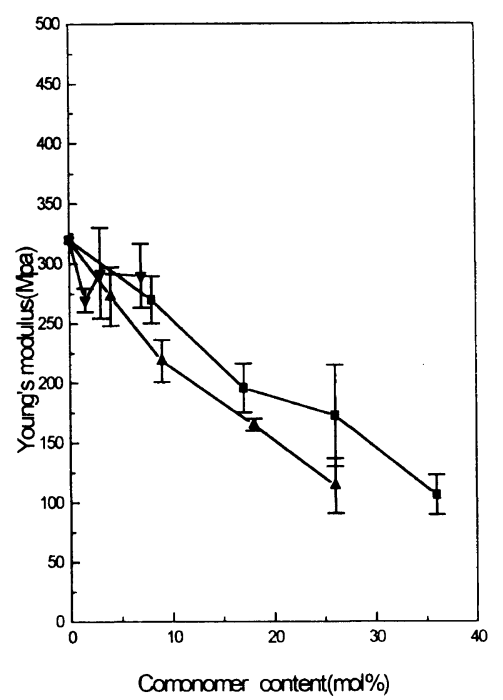

(c)

Figure 4. Mechanical properties of copolyesters with comonomer contents in copolyesters: (a) stress at break, (b) strain at break, (c) Young's modulus; - - - poly(BS-co-ES); - $\mathbf{-}-$, poly(BS-co-PS); - - , poly(BS-co-BeS).

copolymers. Poly(BS-co-ES) series showed relatively higher values than poly(BS-co-PS)s as contents of the secondary repeating unit increased, which may be regarded as an evidence of partial isomorphism in strictly linear copolyester. It has been frequently practiced that the degree of crystallinity of thermoplastic is determined by dividing an observed heat of fusion by the theoretical value for a $100 \%$ crystalline polymer. ${ }^{21}$ The theoretical heat of fusion for PBS calculated by group contribution method based on the data from Table 5.5 in Van Klevlen ${ }^{22}$ yields $113.4 \mathrm{~J} \mathrm{~g}^{-1}$. Relative crystallinities thus determined are also available in Table II. Subsequent second heating thermograms yield in slightly lower heat of fusion than the first runs, indicating relatively fast crystallization of these copolyesters except for the ones with higher content of ethylene succinate or propylene succinate.
XRD patterns for poly(BS-co-ES)s, poly(BS-co-PS)s, and PBS are shown in Figure 3. The diffraction pattern of PBS showed peaks at 2-theta of $18.8^{\circ}, 21.6^{\circ}$, and $22.5^{\circ}$ assigned to the [020], [021], and [110] diffraction peaks. ${ }^{5}$ The copolymerization has effectively diminished the order in the [021] and [110] phase but relatively sharp diffraction pattern was maintained at 2-theta of $18.8^{\circ}$.

\section{Mechanical Properties}

A melt pressed PBS $\left(M_{n}=62000 \mathrm{~g} \mathrm{~mol}^{-1}\right.$ by GPC) film yields tensile strength and Young's modulus around $23 \mathrm{MPa}$ and $320 \mathrm{MPa}$. On the other hand, ultimate strain was in the range of $200-250 \%$, which is significantly lower than that of low density polyethylene (LDPE). This relatively stiff characteristics of PBS may be due to its high crystallinity and large spherulites. One of the objectives of this study was to optimize the chemical 


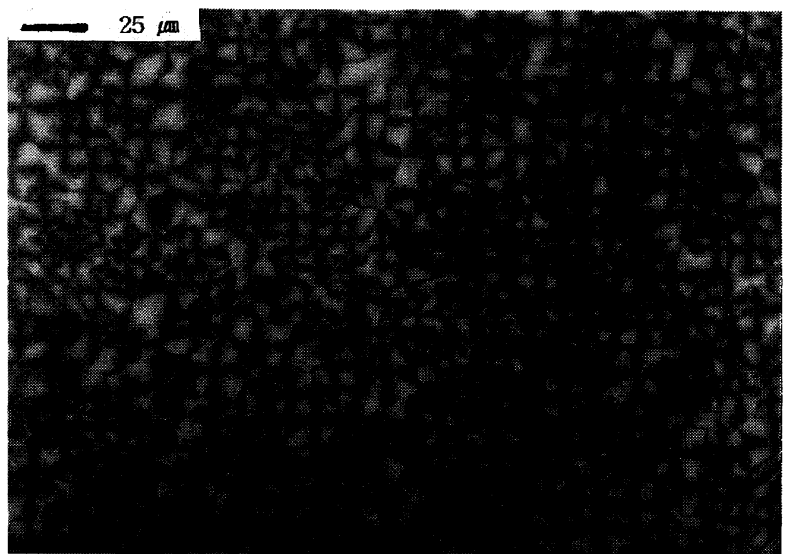

(a)

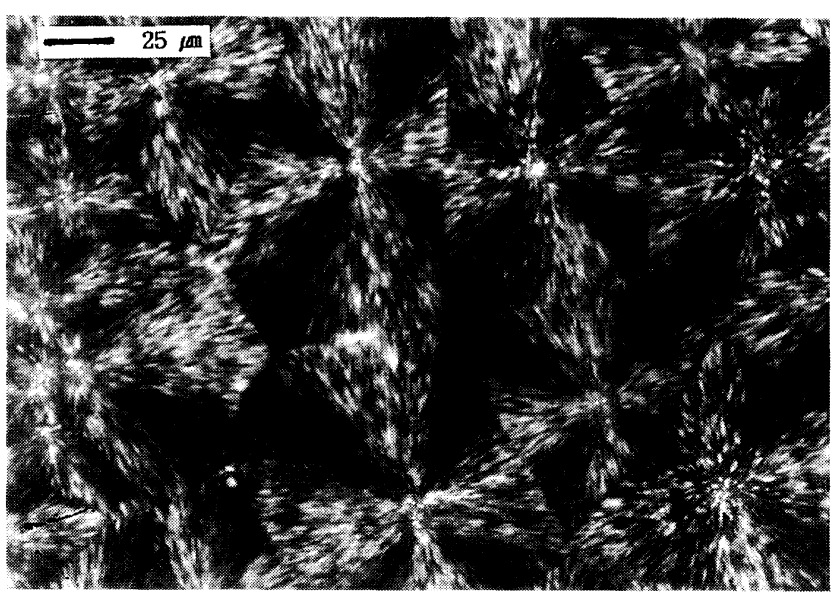

(b)

Figure 5. Polarizing optical micrographs: (a) poly(BS-co-ES)-26, (b) poly(BS-co-PS)-26.

structure and composition of PBS based copolymer to give the critical physical properties, including softening temperature, ultimate strain, and tensile strength in acceptable ranges.

As shown in Figure 4, where the effects of composition on the mechanical strength are given, even enhanced ultimate tensile strength was measured up to $18 \mathrm{~mol} \%$ of ES and PS units in poly(BS-co-ES) and poly(BS-coPS), respectively. It is clear that the incorporation of secondary species to PBS has favorably affected the ultimate strain, which subsequently yields higher ultimate tensile strength. The tensile strength of polyesters used in this study has significantly higher tensile strength than LDPE. It was pointed out earlier by Biggs et al. ${ }^{23}$ that the high tensile strength of crystalline polyester is obtained as a result of the attractive force between polar groups. It was also reported in the same literature that copolyesters may achieve high degree of ordering upon stretching especially when single dibasic acid is used in combination with mixed diols. The substantial increase in tensile strength of copolyesters with ES or PS unit up to $c a .20 \mathrm{~mol} \%$ may be due to the strain induced crystallization. But the orderly repetition of the unit structure is disrupted with further addition of secondary units and tensile strength falls as postulated by Biggs et al.

The ultimate strain has increased drastically with content of ES or PS unit in poly(BS-co-ES) and poly-

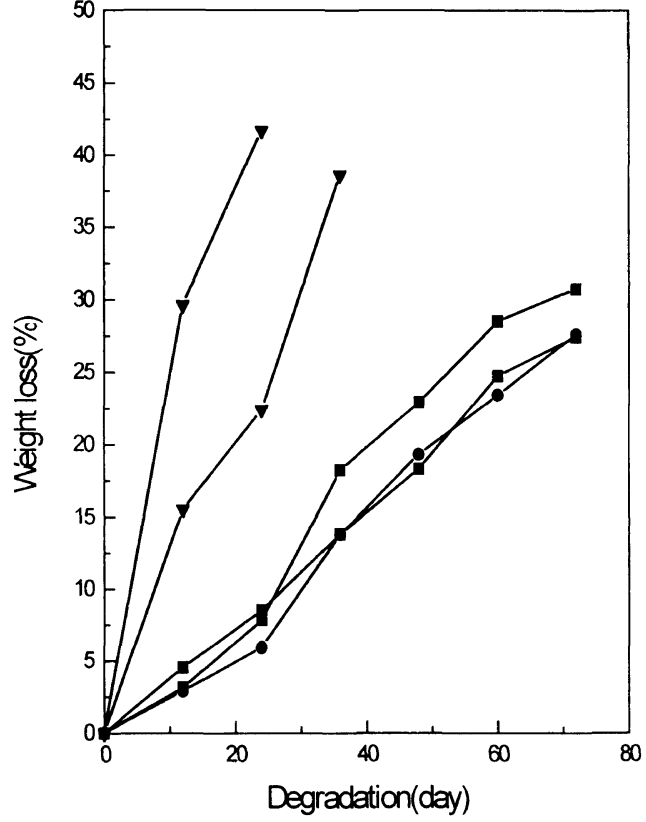

Figure 6. Degradation profile of poly(BS-co-ES) film immersed in alkali aqueous solution of $\mathrm{pH} 12$ at $35^{\circ} \mathrm{C}$ as a function of time: - PBS homopolymer; - ES)-17; - - , poly(BS-co-ES)-26; - -, poly(BS-co-BS)-36.

(BS-co-PS). But some difference in the ultimate strain was observed at $26 \mathrm{~mol} \%$ of minor component for these two series, which may be related to the size of spherulite as observed in the polarizing optical microscopy. Figure 5 shows the spherulites in thin films of poly(BS-co-PS)26 and poly(BS-co-ES)-26 prepared by fusion and air cooling between cover slip and glass slide. In the micrographs poly(BS-co-PS) spherulites exhibit the typical appearance of isotactic polypropylene showing a radiating array of intermingled areas of positive/ zero/negative birefringence crystallized particularly at a small degree of undercooling. ${ }^{24}$ The spherulites of poly(BS-co-PS)-26 have less pronounced Maltese Cross and are larger than those of poly(BS-co-ES)-26. This may be attributable to the lower crystalline melting point of the former by $12 \mathrm{~K}$ and, in turn, smaller undercooling and slower nucleation rate provided that both polymers crystallized at the same temperature.

In poly(BS-co-BeS)s a significant loss in modulus was seen at merely $1.5 \mathrm{~mol} \%$ of $\mathrm{BeS}$ as an indicative of strong disturbance of molecular ordering by introducing cis configuration in the polymer chain. The ultimate strain increased up to $3 \mathrm{~mol} \% \mathrm{BeS}$ units and then leveled off. It appears that tensile strength goes through a maximum with $\mathrm{BeS}$ content, which is analogous to the behavior of rubbery polyesters with extent of vulcanization. This may indicate a topological change from linear to lightly crosslinked structure by the cross polymerzation using the unsaturated moieties in butyne succinate units.

\section{Degradation Study}

The hydrolytic degradation of PBS and copolymers has been investigated on the compression molded films of $0.3 \mathrm{~mm}$ thickness immersed in $\mathrm{NaOH}$ aqueous solutions of $\mathrm{pH} 12$ at $35^{\circ} \mathrm{C}$ employing a shaking incubator. Erlenmayer flasks containing polymer films were gently shaked in swirling mode at $c a .45-50 \mathrm{rpm}$. It has been 


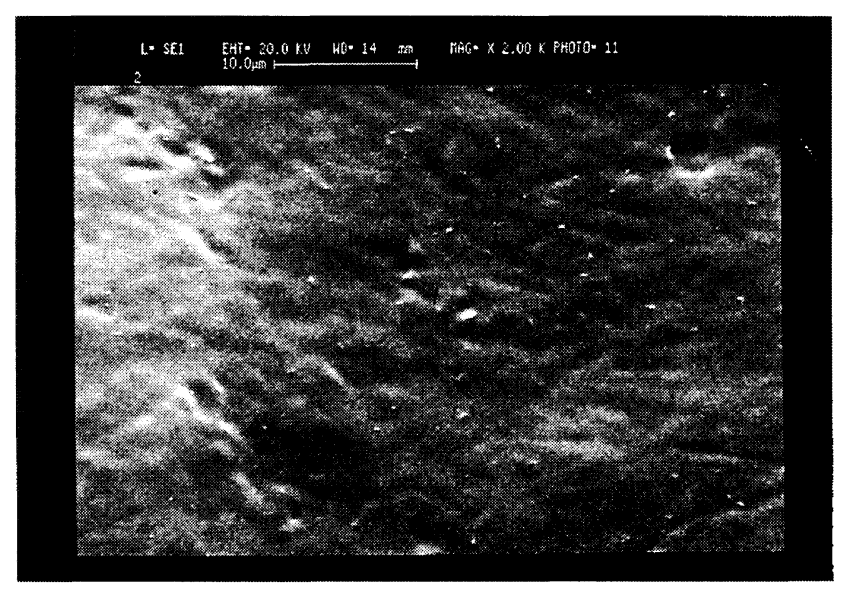

(a)

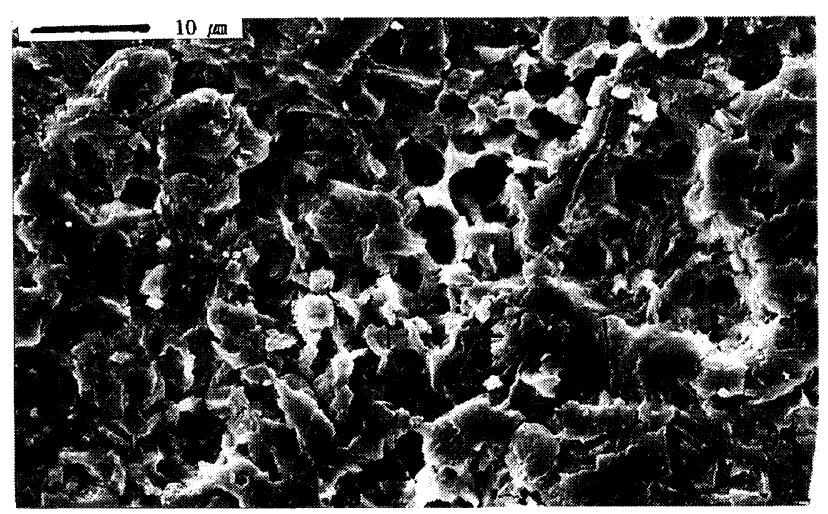

(c)

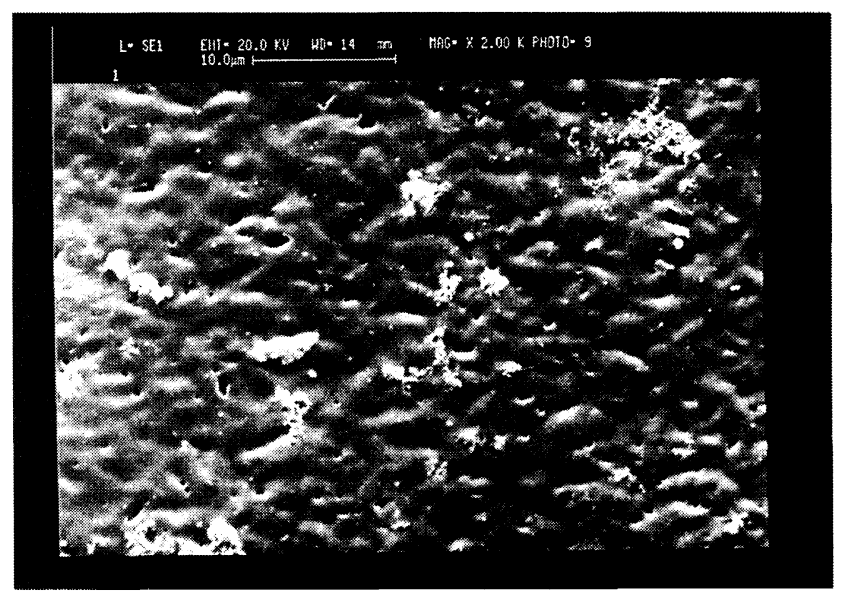

(b)

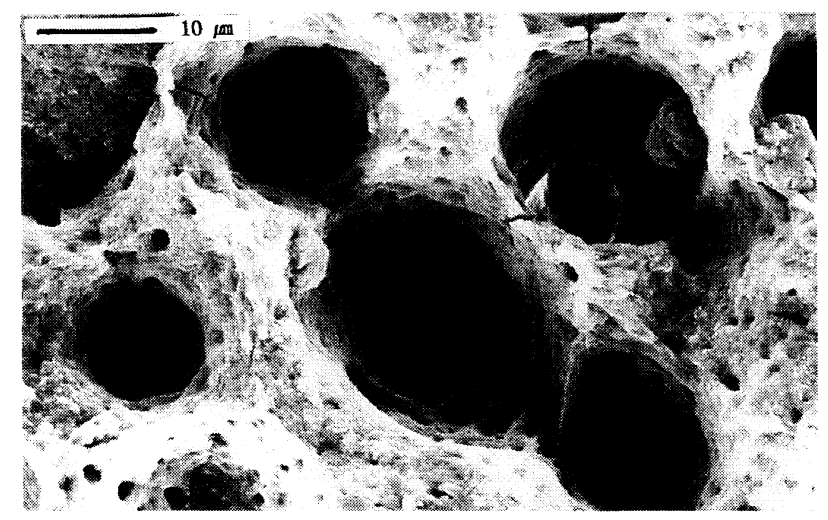

(d)

Figure 7. Scanning electron micrographs of polymer surfaces upon exposure to $\mathrm{NaOH}\left(\mathrm{pH} 12\right.$ ) alkali medium at $35^{\circ} \mathrm{C}$; $\mathrm{PBS}$ after 5 days (a), poly(BS-co-ES)-26 after 5 days (b), PBS after 30 days (c), and poly(BS-co-ES)-26 after 30 days (d).

well received that degradation of aliphatic polyesters derived from the condensation polymerization of diols and dicarboxylic acids, in general, proceeds by hydrolysis with or without the presence of enzyme. For a quick estimation of comparative hydrolytic degradability and reproducible data alkali aqueous medium were often used. The results are shown in Figures 6, 8, and 9, where the weight loss was expressed as a percentage of initial weight.

In the degradation of poly(BS-co-ES) series as plotted in Figure 6 for a period of 75 days the degradation rate was not greatly varied at $8 \mathrm{~mol} \%$ of ethylene succinate although crystallinity has been somewhat diminished. As a result of more dipole-dipole interaction arising from the ethylene succinate unit, less free volume in amorphous regions could have restricted the permeation of nucleophiles and thus compensate the reduction in crystallinity. However, accelerated degradations were observed on the poly(BS-co-ES)s beyond this composition due to more substantial loss of crystallinity as the randomness increased. Figure 7 shows the scanning electron micrograghs of the polyester films in the course of degradation. At the early stage of degradation PBS film maintained a relatively smooth surface, however, a rough surface was developed at the later stage. Generally, chain scissions take place preferentially in amorphous regions to a certain depth by the permeation of nuclephiles and thus hydrated fragments are easily detached from the surface under shear stress. For poly(BS-co-ES)-
26 it was found small pores were produced in several days, which were enlarged as the degradation proceeded. It appears that extremely short chains are more abundantly present in dispersed phase in this copolyester because of low reactivity of half-esterified ethylene glycol, which could be easily scissioned to water soluble species and extracted to the solution leaving fine pores.

The rate of degradation monitored on poly(BS-co-PS) series revealed a composition dependency. As presented in Figure 8 the degradation rate decreased with increasing propylene succinate content up to $9 \mathrm{~mol} \%$. Then, the degradation rate began to rise with further increase in propylene succinate units. Poly(BS-co-PS)-18 exhibited comparable degradation rate to PBS and poly(BS-coPS)-26 exceeded the PBS. The presence of propylene succinate units may promote the hydrophobic nature of polyester, which would negatively influence the hydrolytic susceptability by sterically hindering the access of nucleophiles. In the meantime, the introduction of secondary structure should render polyester more amorphous and less resistant to the nucleophilc attack. As indicated in Figure 8 steric factor seems predominant over reduction of crystallinity at low content of propylene succinate unit and these two factors appear to be equally effective at $18 \mathrm{~mol} \%$ of propylene succinate unit in the copolymer. With further increase in PS content the loss of crystallinity become more critical than the steric hinderance in the degradation. Figure 9 shows the degradation behavior of poly(BS-co-BeS). In contrast to 


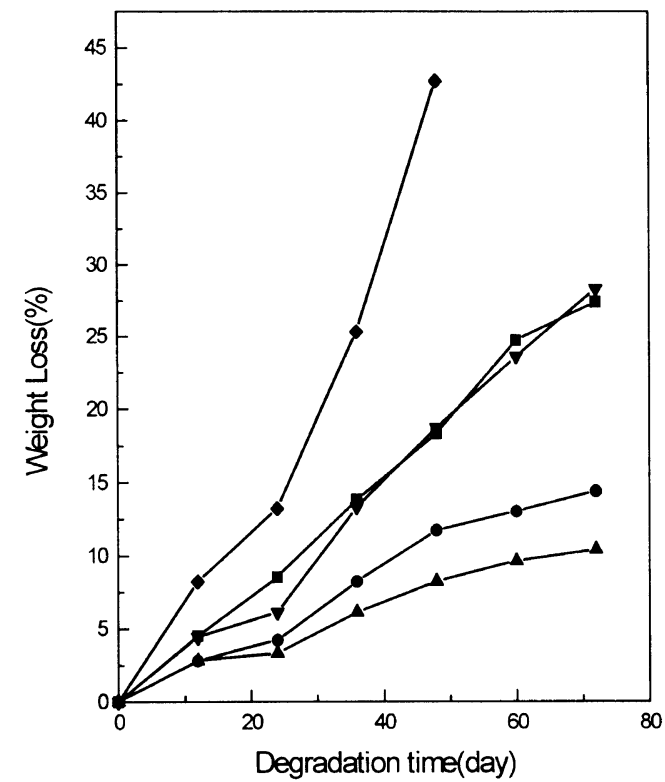

Figure 8. Degradation profile of poly(BS-co-PS) film immersed in alkali aqueous solution of $\mathrm{pH} 12$ at $35^{\circ} \mathrm{C}$ as a function of time: - - ,

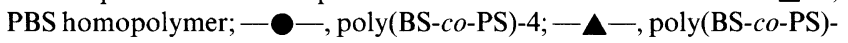
9; - - , poly(BS-co-PS)-18; ——; poly(BS-co-PS)-26.

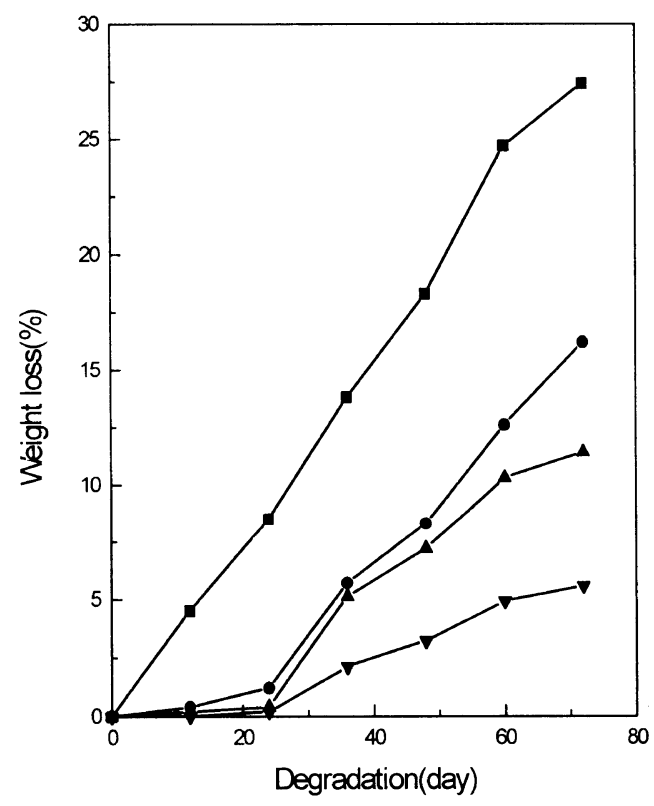

Figure 9. Degradation profile of poly(BS-co-BeS) film immersed in alkali aqueous solution of $\mathrm{pH} 12$ at $35^{\circ} \mathrm{C}$ as a function of time: - - PBS homopolymer; - - poly(BS-co-BeS)-1.5, - - - poly(BS-co$\mathrm{BeS})-3 ;-\nabla-$, poly(BS-co-BeS)-7.

poly(BS-co-ES) series butyne succinate unit has an adverse effect on the degradation rate. Degradation rate of this series has decreased consistently with increasing content of BeS. Furthermore, this series of copolymers showed lag times where little weight loss occurred followed by an onset of degradation. This retarded degradation behavior may be ascribable to the topo- logical change from linear to branched and/or lightly crosslinked species as discussed earlier.

\section{CONCLUSIONS}

The structural modifications of PBS via copolymerization using various diols have demonstrated the possibility of control of degradation rate as well as improving the ductility of the homopolymer. The hydrophobicity and topological variation of polyesters were seen to have significant effects on the degradation rate of polyester. It was shown that at low content of propylene succinate unit steric factor dominates over crystallinity effect. Moreover, remarkable is the fact that the incorporation of unsaturated diol by only a few percent yields a lag time on degradation in basic aqueous medium.

Acknowledgments. The authors wish to thank the Korea Science and Engineering Foundation for their financial support (Grant No. 95-0300-07-03-3).

\section{REFERENCES}

1. S. J. Huang, in "Encyclopedia of Polymer Science and Engineering," H. F. Mark, N. Bikales, C. G. Overberger, and G. Menges Ed., Wiley, New York, N.Y., 1985.

2. P. P. Klemchuk, Polym. Degrad. Stab., 27, 183 (1990).

3. J. Dahlmann, G. Rafler, K. Fechner, and B. Mehlis, Brit. Polym J., 23, 235 (1990).

4. V. V. Korshak and S. V. Vinogradora, "Polyester," Pergamon Press, New York, N.Y., 1965, p 41.

5. K. J. Ihn, E. S. Yoo, and S. S. Im, Macromolecules, 28, 2460 (1995).

6. E. Takiyama and T. Fujimaki, Plastics, 43, 87 (1992).

7. W. Taniguchi, Polym. Prepr., Jpn., 42, 3787 (1993).

8. Y. Tokiwa, Plastic Age, June, 166, (1993).

9. A. C Albertsson and Ljungguist, J. Macromol. Sci. Chem., A23, 393 (1986).

10. W. L. Chang and T. Karalis, J. Polym. Sci., Part A: Polym. Chem. Ed., 31, 493 (1993).

11. D. K. Gilding and A. M. Reed, Polymer, 20, 1459 (1979).

12. G. J. Howard and S. Knutton, Polymer, 9, 527 (1969).

13. T. Kanamoto, J. Kuwano, and K. Tanaka, Rept. Prog. Polym. Phys. Jpn., 16, 187 (1973).

14. Y. Saito, S. Nakamura, M. Hiramitsu, and Y. Doi, Polym. Int., 39, 169 (1996).

15. D. K. Kim, Y. S. Shin, S. S. Im, Y. Yoo, and J. R. Haw, Polymer (Korea), 20, 451, (1996).

16. J. Yamanis and M. Adelman, J. Polym. Sci., Polym. Chem. Ed., 14, 1945 (1976).

17. G. Odian, "Principles of Polymerization," 2nd ed., John Wiley \& Sons, New York, N.Y., 1981, p 60.

18. R. Yamadora and M. Murano, J. Polym. Sci., Part A-1, 5, 2259 (1967).

19. L. L. Zhu and G. Wegner, Makromol. Chem., 182, 3625 (1981).

20. J. Brandrup and E. H. Immergut, "Polymer Handbook," 3rd ed, John Wiley \& Sons, New York, N.Y., 1989, Chapter IV.

21. M. Dole, J. Polym. Sci., Part C, 18, 57 (1967).

22. D. W. Van Krevelen, "Properties of Polymers," 2nd ed, Elsevier Scientific, Amsterdam, 1976.

23. B. S. Biggs, R. H. Erickson, and C. S. Fuller, Ind. Eng. Chem., 39, 1090 (1947).

24. D. R. Norton and A. Keller, Polymer, 26, 704 (1985). 